\title{
Role of traditional medicine astragalus membranaceus in the treatment of nephritis (10 cases)
}

\author{
Zhu G* \\ The Institute of Oncology, Tehran University of Medical Sciences, Tehran, Iran
}

\begin{abstract}
Objective: To study the long-term effect of Astragalus on urine protein in patients with nephrotic syndrome.

Method: 10 available patients were collected during 1991 to 2017.All 10 patients were treated using prednisone and traditional medicine (TCM) containing Astragalus. Two patients were, in addition, placed on immunosuppressive drugs cyclophosphamide and nitrogen mustard injection.

Results: All 10 patients had successfully treated with TCM containing astragalus and corticosteroid therapy. These included improved clinical symptoms and obviously decreased proteinuria. One glomerulonephritis was, at relapse, receiving TCM Astragalus only. In this trick case, he seemed to have the nephrotic syndrome with severe edema, hypoproteinemia, hypercholesterolemia and massive proteinuria. Another membrano proliferative glomerulonephritis associated with anaphylactatic purpura glomerulonephritis had therapeutical improved benefits following TCM and a small dosage of dexamethasone. The disease-free survival time were $25,24,24,23,23,8,17,13$ years respectively.
\end{abstract}

Conclusion: Astragalus has obvious improved effect on renal diseases especially the patient with nephrotic syndrome, presentation of decreasing urine protein and increasing the plasma protein. TCM Astragalus provide another promising strategy in addition to corticosteroid therapy

\section{Introduction}

Astragalus is a large genus of about 3,000 species of herbs and small shrubs, belonging to the lugume family Fabaceae and the subfamily Faboideae. The genus is native to temperate regions of the Northern Hemisphere. Common names include milkvetch (most species, Figure 1), Iocoweed (in North America, some species). The chemical constituent astragaloside IV and cycloastragenol is being studied to help combat infections associated with chronic diseases and aging. High-quality clinical trials in people are generally lacking. There is some preliminary evidence to suggest that astragalus, either alone or in combination with other herbs, may have potential benefits for the immune system, heart, liver and kidney, and as an adjunctive therapy for cancer. In this paper, I reported respectively astragalus in a long-term study of glomerulonephritis and associated with nephrotic syndrome.
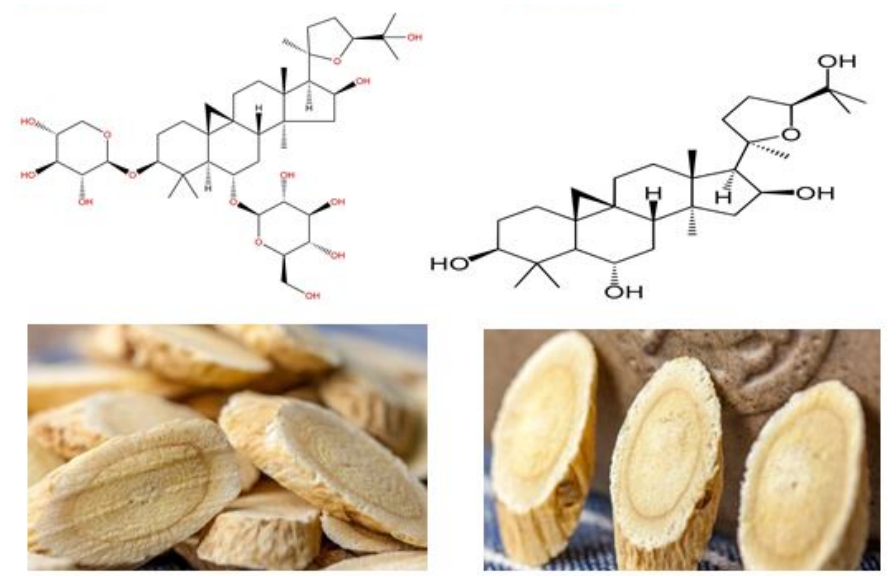

Figure 1. The chemical structure of astragaloside IV (left) and cycloastragenol (right).

\section{Materials and methods}

10 patients with available renal diseases were entered in the study during 1991-2017.The sex ratio of male: female was 6:4 respectively. The range age at onset was 4.5-52 years. The clinical diagnoses of the diseases were nephrotic syndrome 6 cases, glomerulonephritis 3 cases and 1 anaphylactatoid purpura glomerulonephritis. All patients were used with corticosteroid therapy and traditional medicine (TCM) containing Astragalus.

\section{Results}

During the schedule of drug administration, 10 patients obtained complete remission and/or partial remission through different dosage of combination therapy of corticosteroid and TCM. After treatment, urine analysis showed the results that proteinuria was declined to trace or negative in 3 cases, decreased to $1+$ proteinuria in 2 cases, $2+$ proteinuria in 3 cases. The therapeutical courses was varied from 10 days to 4 months. The detail data was summarized in Table 1.

\section{Case reports}

A 41/2-year-old boy presented with a history of nephrotic syndrome on July 3,1991 due to recurrent episode of progressively increasing pedal edema extending to involve both thighs and his scrotum.He also experienced swelling around his eyes and his severe facial edema. There

${ }^{*}$ Correspondence to: Zhu G, Institute of Oncology of George Zhu, Beijing, China, E-mail: sansan4240732@163.com

Key words: nephrotic syndrome, corticosteroid, astragalus

Received: May 07, 2018; Accepted: May 21, 2018; Published: May 24, 2018 
Table 1. Patients characteristics

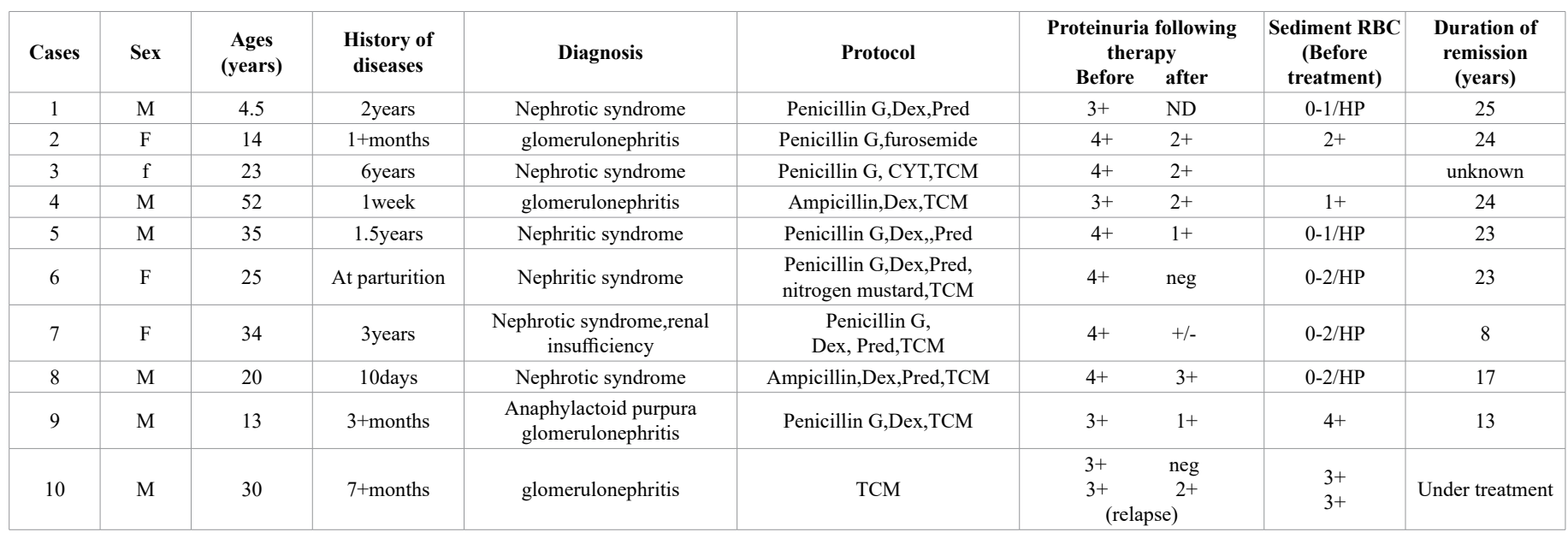

was edema in his upper extremities. Ascites++++. There was a past history of similar episodes for the disease in another hospital four times without much efficacy. Urinalysis: proteinuria 3+; sediment showed 0-1 RBC/HP. Treatment consisted of Penicillin and the therapeutic dosage of corticosteroid (dexamethasone) therapy. And later oral prednisone at gradual dose was to be maintained for near 1 year. In a 25 years of follow up, he is well.

A 14-year-old girl was admitted for presentation of 1 month of both lower extremities edema and ascites++, accompanied with bilateral flank pain on September 10,1993. BP80/40mmHg. Urinalysis showed $2+\mathrm{RBC}$ and $4+$ proteinuria. The diagnosis of acute glomerulonephritis was considered. Treatment consisted of penicillin in full dose with furosemide injection (up to $110 \mathrm{mg}$ ). With edema and ascites disappearance, repeat urinalysis showed $0-2 \mathrm{RBC} / \mathrm{HP}$ and 2-3 WBC/ $\mathrm{HP}$ with $2+$ proteinuria. As an outpatient, she continued to penicillin. A 24-years of follow up she was well.

A 23-year-old woman was referred to the relapse of her nephrotic syndrome on December 23,1993. BP90/70mmHg. Urinalysis: protein $4+$. She was given penicillin G sodium, hydrochlorothiazide, and oral prednisone, with adjuvant traditional medicine. Repeat urinalysis showed $2+$ protein. She was once admitted another hospital and urine protein negative following cyclophosphamide (CYT) therapy.

A 52-year-old man was admitted to the hospital on April 9,1994. He was complaint of thirsty and kidneys palpable pain of 4 days duration. At a routine physical examination, there was revealed a percussion of bilateral flank pain. No periorbital or pedal edema was noted. BP60/45mmHg. Urinalysis showed $3+$ protein and $1+\mathrm{RBC} /$ HP. The diagnosis of glomerulonephritis was considered. He was given ampicillin and dexamethasone intravenously, with traditional medicine. Repeat urine analysis was 3-6 $\mathrm{RBC} / \mathrm{HP}$ with $2+$ proteinuria. The traditional medicine was continuous to be taken for a period of time. Traditional medicine consisted of Astragalus membranaceus, Codonopis pilosula,Ophiopogon japonicus kergawl, rehmannia, red-rooted salvia, Poria cocos, rhizoma anemarrhena, rhizoma imperata. A 24-years follow up he remained well.

A 35-year-old man was admitted to the hospital on December 8,1994 because of his relapse of nephrotic syndrome. In February,1993, he was complaint of facial edema. Urine analysis: proteinuria 4+; sediment- $0-1 \mathrm{RBC} / \mathrm{HP}$. He was placed on penicillin G sodium and dexamethasone intravenously. Later, oral prednisone at gradual dose accordingly was to be taken. At discharge, repeat urine analysis showed 1+ proteinuria and 0-1 RBC/HP. A 23years follow up he was well.

A 25-year-old woman was admitted to the hospital on March 15,1995 because of her nephrotic syndrome. She developed generalized severe edema at parturition. At physical examination, on admission, revealed that the abdomen was a markedly protuberant like a balloon like appearance. Ascites++++. Two plus pitting edema was present bilaterally from pedal extending to involve both thighs. BP90/60 $\mathrm{mmHg}$. Laboratory data: Hemogram: hemoglobin was $110 \mathrm{~g} / \mathrm{l}$,WBC $8.8 \times 109$ / l,plt 230x109/l. Urinalysis: protein 4+;sediment:0-2 RBC/HP and 0-6 WBC/HP. The diagnosis of nephrotic syndrome was made. Treatment consisted of penicillin G sodium and dexamethasone intravenously iv drop, intravenous nitrogen mustard, and with traditional medicine. On April 8, repeat urine analysis showed $2+$ proteinuria. And, the traditional medicine (TCM) and oral prednisone $(40 \mathrm{mg} / \mathrm{d})$ was continued to be undergoing. Repeat urine analysis on April 2,1996 showed protein negative. On October 2,1996 the TCM was given again due to the relapse of 4+ proteinuria, and on October 23, urine protein was declined to 2+. TCM consisted of Astragalus membranaceus, rehmannia, rhizoma dioscoreae,poria cocos,rhizoma alismatis,red-rooted salvia,rhizoma anemarrhena,polyporus,peach kernel,asiatic plantain seed,lycium chinenses mill,fructus corni,paeonia suffruticosa,orange peel. A 23years follow up, she was well.

A 34-year-old woman was admitted to the hospital on August 21,1996 due to her nephrotic syndrome and renal insufficiency for 3 years. She was complaint of pallor, fatigue, facial edema. At physical examination, she had a percussion of bilateral flank pain. Ascites +++. BP120/80mmHg. Urine analysis: protein 4+; sediment- 1-2 RBC/ $\mathrm{HP}$ and 4-6 WBC/HP. Blood chemistry showed BUN $7.7 \mathrm{mmol} / \mathrm{l}$ (control:2.9-7.1 mmol/l). She was given penicillin $\mathrm{G}$ sodium and dexamethasone intravenously, with traditional medicine (TCM) and adjuvant intramuscular testosterone. On August 29,1996, proteinuria was declined to $1+$. The TCM and testosterone were continued to be taken later. On September 8 and October 24,1996 respectively, repeat urinalysis showed protein negative. She was 8 years survivor.

A 20-year-old man was admitted to the hospital because of his nephrotic syndrome on August 24,2001. At physical examination revealed edema involving his face and his lower extremities. Ascites ++. BP105/75mmHg. Laboratory data: Hemogram: Hemoglobin is $133 \mathrm{~g} / \mathrm{l}$, WBC $6.9 \times 109 / 1$, plt $303 \times 109 / 1$. Urinalysis: protein $4+$; 
sediment:1 granular cast. Treatment consisted of Ampicillin sodium and corticosteroid therapy, with adjuvant traditional medicine (TCM). Repeat urinalysis at discharged hospital on September 3 showed 3+ proteinuria and $0-2 \mathrm{RBC} / \mathrm{HP}$. As an outpatient, he continued to TCM. In the follow up the patient with 17 years of disease-free survival remained well.

A 13-year-old boy presented with a history of a sudden onset of arthralgia, skin rash, abdominal pain, 3+ months before being seen on March 21,2004 . BP $90 / 60 \mathrm{mmHg}$. He was admitted to another hospital on December 30,2003 , at that time, he was thought to have the diagnosis of anaphylactatic purpura glomerulonephritis. Proteinuria 4+ was declined to $3+$ following prednisone $(40 \mathrm{mg} /$ day). Later, his proteinuria was varying degree from $2+$ to $3+$ after using prednisone $(30 \mathrm{mg} /$ day) in a transferred provincial hospital. A renal biopsy was the most likely diagnosis of membrano proliferative glomerulonephritis. He was immediately to the emergency owing to naked hematuria. Urinalysis was continued to show $4+\mathrm{RBC}$ and $3+$ protein. Treatment consisted of penicillin $G$ sodium and a small dosage of $5 \mathrm{mg}$ dexamethasone intravenously, and etamsylate injection intensively staunched the bleeding of urine. During hospital, TCM was also taken. Repeat urinalysis, at discharge, showed 2+-3+ $\mathrm{RBC}$ and $1+$ proteinuria. As an outpatient, he was placed on TCM investigation. TCM consisted of Astragalus membranaceus, rehmannia, $r$ ehmannia glutinosa preparatae,ophiopogon japonicus kergawl,red-rooted salvia,parasitic loranthus,fructus corni,rhizoma anemarrhena,paeonia suffruticosa,asiatic plantain seed,herba cirsil,herba eclipta,rhizoma imperata.

A 30-year-old man was diagnosed as having glomerulonephritis on April 22, 2017. He had a history of facial and lower extremity edema for $7+$ months, accompanied with thirsty and fatigue. At physical examination, on admission, the abdomen was a moderate protuberant and a percussion of bilateral flank pain. Ascites ++ . Two plus pitting edema was present bilaterally from pedal extending to involve both knees. BP130/85mmHg. Urine analysis showed 3+ RBC and $3+$ protein. He was placed on the main traditional medicine (TCM) and a small dosage of oral prednisone. Repeat urine analysis on July 2 to 24 showed RBC $1+$ and proteinuria $1+$ to trace, and on September 3 urinalysis showed protein negative. On December 7,2017, he developed a relapse of similar episodes for the disease owing to catching a cold. Urine analysis disclosed $3+\mathrm{RBC}$ and $3+$ proteinuria. Blood chemistry revealed that total protein was 30.5g/l (control:60$80 \mathrm{~g} / \mathrm{l}$ ), serum albumin $12.3 \mathrm{~g} / \mathrm{l}$ (control:35-55g/l), serum globumin 18.2g/l (control:25-30g/l). Serum cholesterol $12.8 \mathrm{mmol}$ (control:2.23-5 $\mathrm{mmol}$ ), serum TG $4.13 \mathrm{mmol}$ (control:0.56-2.3 mmol), serum LDL-C $9.49 \mathrm{mmol}$ (control:1.3-3.37 $\mathrm{mmol}$ ). Her edema had disappearance following TCM. In March,2018 the third relapse was noted because of recurrent episode of generalized edema. Urinalysis: protein 3+, RBC 2+; sediment: 0-1 granular cast/HP.TCM was continued to be given. TCM consisted of Astragalus membranaceus, codonopsis pilosula, rehmannia, rehmannia glutinosa preparatae, poria cocos, rhizoma alismatis, asiatic plantain seed,rhizoma anemarrhena,red-rooted salvia, rhizoma dioscoreae,parasitic loranthus, fructus corni, houttuynia cordata thunb, herba leonuri,lycium chinenses mill,herba cirsil,rhizoma imperata. On April 16, 2018, urine analysis showed 2+ proteinuria and 2+ RBC. At present, he was undergoing TCM investigation.

\section{Discussion}

In this study,ten renal diseases associated with nephrotic syndrome had successfully treated using combination therapy of corticosteroids and TCM containing Astragalus. The data provide astragalus in clinical therapeutical benefits in nephrotic syndrome. Astragalus was value to be recommended as adjuvant use.

Membranous glomerulonephritis is characterized by normal serum complement level, nonselective proteinuria, lipiduria, hematuria, deposition of IgG and complement in the glomerular basement membrane (giving a characteristic spiked appearance to the basement membrane) [1,2] and the nephrotic syndrome. In membranoproliferative glomerulonephritis associated nephrotic syndrome, representing an immune complex disease, complement degradation is activated by a factor at the $\mathrm{C} 3$ level and depresses $\mathrm{C} 3$ in the serum. C4, being a step earlier, may be normal [3]. In this group, case 10, membranoproliferative glomerulonephritis is likely considered because of RBC amounts in his urine. In the absence of more conclusive finding, the correct diagnosis could only be reached by combining all of the clinical and pathological facts such as a kidney biopsy.

Steroid hormones (prednisone) produce complete remission in over half (60\%) of the patients treated, and partial remission in another $22 \%$ [4]. Immunosuppressive drugs such as cyclophosphamide (Cytoxan) and nitrogen mustard are useful in steroid failure or in recurrent attacks. The administration with Cytoxan and mustard after steroids in case 3 and 5 in my group and others seems to be of some value $[5,6]$.

\section{Conflicts of interest}

The authors declare that there is no conflict of interest regarding the publication of this paper.

\section{References}

1. McCluskey RT, Vassalli P, Gallo G, Baldwin DS (1966) An immunofluorescent study of pathogenic mechanisms in glomerular diseases. N Engl J Med 274: 695-701. [Crossref]

2. Lange K, Treser G, Sagel I, Ty A, Wasserman E (1966) Routine immunohistology in renal diseases. Ann Intern Med 64: 25-40. [Crossref]

3. Cameron JS, Ogg CS, White RH, Glasgow EF (1973) The clinical features and prognosis of patients with normocomplementemic mesangiocapillary gliomerulonephritis. Clin Neph 1: 8 [Crossref]

4. Habib R, Kleinknecht C (1971) The primary nephrotic syndrome of childhood Classification and clinicopathologic study of 406 cases. Pathol Annu 6: 417-474. [Crossref]

5. Etteldorf JN, Roy S, Summitt RL, Sweeney MJ, Wall HP, et al. (1967) Cyclophosphamide in the treatment of idiopathic lipoid nephrosis. J Pediatr 70: 758-766. [Crossref]

6. Moncrieff MW, White RH, Oggs CS, Cameron JS (1969) Cyclophosphamide therapy in the nephrotic syndrome in childhood. Br Med J 1: 666-671. [Crossref]

Copyright: (C2018 Zhu G. This is an open-access article distributed under the terms of the Creative Commons Attribution License, which permits unrestricted use, distribution, and reproduction in any medium, provided the original author and source are credited. 